\title{
Intrauterine transplantation of autologous menstrual blood stem cells in the treatment of refractory intrauterine adhesion: a prospective cohort study
}

Hailan Ma

Guiping People's Hospital

Mengting Liu

Wuhan Hmilaton Biotechnology

Dongcheng Wu ( $\sim$ bcdcwu@hotmail.com)

Wuhan University https://orcid.org/0000-0002-2989-7291

Yufeng Li

Huazhong University of Science and Technology Tongji Medical College

Wei Wang

Guiping PeoPle's Hospital

Keqin Yang

Guiping People's Hospital

Lanying Lu

Guiping People's Hospital

Mei He

Guiping People's Hospital

Taoran Deng

Huazhong University of Science and Technology Tongji Medical College

Meiling Li

Guiping People's Hospital

\section{Wei Wang}

Wuhan Hamilton Biotechnology

\section{Research article}

Keywords: Menstrual blood stem cells, autologous transplantation, intrauterine adhesion, endometrial thickness

Posted Date: December 1st, 2019

DOI: https://doi.org/10.21203/rs.2.17776/v1 
License: (c) (i) This work is licensed under a Creative Commons Attribution 4.0 International License. Read Full License 


\section{Abstract}

Background: The loss of endometrial stem cells has been associated with intrauterine adhesion caused by miscarriage, repeated improper intrauterine operation or infection. Cell-based therapies are promising in regenerative medicine. However, the effects of menstrual blood stem cells (MenSCs) therapy on impaired endometrium need to be explored and validated. This study evaluates the effects of intrauterine transplantation of MenSCs on endometrial thickness and pregnancy outcomes in patients with refractory intrauterine adhesion.

Methods: This study included a group of infertile women ( $n=12$, age 22-40 years), with refractory intrauterine adhesion (Grade III - IV). Autologous MenSCs isolated from the women' menstrual blood were expanded in vitro and transplanted into their uteruses, followed by hormone replacement therapy. Transvaginal ultrasound examination was performed to assess the endometrial thickness.

Transabdominal ultrasound was conducted to detect pregnancy outcome.

Results: Autologous MenSCs were successfully isolated and expanded from menstrual blood and transplanted into the uterus of each patient. A significant improvement of the endometrial thickness was observed (from $3.9 \pm 0.9 \mathrm{~mm}$ pre-treatment to $7.5 \pm 0.6 \mathrm{~mm}$ post-treatment $(P<0.001)$ ). No adverse reaction was observed. The duration of menstruation was increased (from $2.4 \pm 0.7$ days pre-treatment to $5.3 \pm 0.6$ days post-treatment $(P<0.001))$. Five out of 12 patients achieved clinical pregnancy.

Conclusions: Intrauterine transplantation of autologous MenSCs results in regeneration of endometrium, a prolongation of menstrual duration and an increase rate of pregnancy in patients with refractory intrauterine adhesion.

\section{Background}

Intrauterine adhesion (IUA), also known as Asherman's syndrome (AS), is caused by miscarriage, repeated improper intrauterine operation or infection and leads to a series of clinical complications, such as hypermenorrhea or amenorrhea, infertility and recurrent loss of pregnancy [1]. The first-line treatments for AS include hysteroscopic adhesiolysis to separate adhesion directly, barrier media to prevent re-adhesion, estrogen and progesterone to stimulate endometrial regeneration. All these conventional therapies are temporary palliative treatment for the symptoms. Furthermore, the efficacy on patients with severe AS is unsatisfactory and the rate of post-treatment re-adhesion is high. Therefore, there is an urgent need to explore new treatment methods [2].

Endometrium includes functionalis and basalis. The functionalis sheds monthly, while the basalis where stem cells settle can renew the endometrium under hormonal influence. Remodeling of endometrial tissue during menses mirrors its potential to renew, which relies on the residing adult stem cells [3]. When repeated injury is inflicted to the endometrium, the number of stem cells becomes depleted. Inflammatory reaction caused by infection can also damage the function of stem cells [4]. The loss or dysfunction of endometrial stem cells is an important contributor to IUA and re-adhesion after curettage. 
Mesenchymal stem cells are widely used in basic research and clinical application. They not only selfrenew and differentiate, but also secret growth, angiogenesis, anti-inflammatory and immunoregulatory factors. Transplantation of autologous bone marrow stem cells has been reported to effectively promote endometrial growth [5]. However, age requirement and the difficulty of collecting the bone marrow have limited the practice of this method. MenSCs have mesenchymal cell-like characteristics with high expressions of CD73, CD105, CD90, CD44 and the absence of hematopoietic stem cells markers CD34 and CD45. They could be induced into adipocytes, osteoblast, cartilage, cardiomyocytes, liver-like, nucleus pulposus-like and glial-like cells [6]. MenSCs therapy showed good results in many animal models related to reproductive system, cardiovascular system, nervous system, digestive system and respiratory system diseases [7-12]. In addition, MenSCs originate from endometrium, which potentially makes them better inhabitants of the injured site in the microenvironment of endometrium [13]. Collection of MenSCs avoids painful procedures and ethical controversies involved in the collection of other stem cells. However, there are few clinical reports about MenSCs treatment for intrauterine adhesion.

Therefore, the effectiveness of MenSCs as a treatment for infertility caused by intrauterine adhesion needs to be explored and validated. Here, our study is designed to further test this therapy for AS and demonstrate the effects of intrauterine transplantation of autologous MenSCs on endometrium thickness, menstrual duration and pregnancy rate.

\section{Methods}

Potential treatment candidates were screened and recruited at the Reproductive Center of the Guiping People's Hospital in Guangxi. The inclusion criteria included: age between 22-40 years, diagnosis of Grade III-IV AS (According to the European Society of Gynecological Endoscopy), less than $6 \mathrm{~mm}$ endometrial thickness after traditional treatment, and more than 1 year of infertility. The exclusion criteria included: infertility caused by congenital or inherited disease and acute urogenital infection and other genital disease.

Menstrual blood was collected on the second day of the menstrual cycle. The MenSCs were isolated, expanded and identified in vitro. On the second day of next menstrual cycle, $1 \times 10^{7}$ well-prepared MenSCs were transplanted into the uterus. Changes of pre-and after-treatment endometrial thickness were monitored by ultrasound. The menstrual duration was recorded pre- and post-treatment. Embryo transfers were performed when the endometrial thickness reached ${ }^{3} 7 \mathrm{~mm}$. Clinical pregnancy rate was recorded after either embryo transfer or nature conception.

\section{MenSCs isolation, identification and preservation}

On the second day of the menstrual cycle, patients came to the hospital. Vulva was disinfected first and a menstrual cup rinsed by heparin sodium solution was placed in the vaginal vault to collect menstrual blood, which was transferred to PBS containing the antibiotic antimycotic. Samples were maintained at $4^{\circ} \mathrm{C}$ until reaching the processing laboratory. 
Mononuclear cells were isolated by Ficoll gradient separation method and cultured in serum-free medium supplemented with Ultroser $\mathrm{G}$ and L-Glutamine. Cells were washed with PBS to remove the non-adherent fraction after fresh medium was added and incubated overnight. The cells were detached by $0.25 \%$ trypsin after reaching $80-90 \%$ confluence and sub-cultured. We collected and preserved the passage four cells. At the same time, the culture supernatant was tested for the presence of bacteria, fungi, mycoplasma and endotoxin. The cells were abandoned upon detection of microbial contamination. Antihuman antibodies for CD73, CD105, CD90, CD34, CD45 and HLA-DR were used to characterize MenSCs. Stained cells were analyzed with Beckman CytoFlex Flow Cytometer.

\section{Differentiation of MenSCs}

MenSCs were seeded at the density of 10,000 cells $/ \mathrm{cm}^{2}$ in 6-well plates and maintained in the growth medium. When cells reached $70-80 \%$ confluence, growth medium was then substituted with the adipogenic or osteogenic inducing medium which was changed every 3-4 days. After 14 days of adipogenic induction and 21 days of osteogenic induction, the cells were washed with PBS, fixed with $4 \%$ paraformaldehyde for 10 minutes and washed with PBS twice. Adipogenic and osteogenic differentiation was confirmed via Oil Red and Alizarin Red staining respectively in combination with real-time qPCR analysis (described below). MenSCs cultured in growth medium were used as a control.

\section{Real-time qPCR}

Total cellular RNA was isolated from adipogenically or osteogenically committed and uncommitted cells by Trizol Reagent. $1 \mu \mathrm{g}$ RNA was reverse transcribed into cDNA. Fluorescence quantitative PCR was performed to detect the expression of markers of adipogenic or osteogenic differentiation (LPL: FTCCAGCTGGACCTAACTTTG, R-AGGGGACCCTCTGGTGAATG; OC: F-ATGAGAGCCCTCACACTCCTC, RGCAGCCTTTGTGTCCAAGCAG; GAPDH: F-TGCACCACCAACTGCTTAGC, RGGCATGGACTGTGGTCATGAG).

\section{Autologous MenSCs transplantation}

On the second day of the menstrual cycle, patients were asked to be prepared for transplantation. MenSCs were thawed in a $37^{\circ} \mathrm{C}$ water bath, washed twice, counted and suspended in physiological saline. Patients were held in a lithotomy position. Vulva and vagina were disinfected with iodophor, vagina, cervix and vaginal fornix were wiped with sterile gauze, blood clot in the uterine cavity was cleared, and the endometrium was scraped slightly before transplantation. Under the guidance of transvaginal ultrasound, an embryo transfer tube was inserted into the cervix to reach the basal layer of endometrium. The inner cannula was removed and attached to a syringe filled with $1 \mathrm{ml}$ MenSCs suspension. $0.5 \mathrm{ml}$ suspension was slowly injected, and then the transplantation tube was withdrawn while the remaining $0.5 \mathrm{ml}$ solution was continuously injected until the tip was fully withdrawn from the endometrium. This process was repeated at 2 additional sites. Patients were discharged after the stem cell transplantation after 2 hours of observation. 


\section{Hormone replacement therapy (HRP)}

Starting on the day of the transplantation, Femoston red tablets (Abbott, Netherlands) were administered orally 3 times a day for 14 days. If the endometrium thickness was more than $7 \mathrm{~mm}$ after 14 days, Femoston yellow tablets $(2 \mathrm{mg})$ were administered orally 3 times a day for 14 days. On the third day after withdrawal bleeding, patients continued to take Femoston red tablets orally for 12 more days. If the thickness was still ${ }^{3} 7 \mathrm{~mm}$, peripheral blood was examined for HCG.

If the endometrium thickness was less than $7 \mathrm{~mm}$ after taking Femoston red tablets for 14 days, the patients continued to take Femoston red tablets for another 3 days. If the subsequent examination still shows less than 7-mm-thick endometrium, the patients would switch to Femoston yellow tablets for 14 days. On the third day after withdrawal bleeding, this hormone therapy was repeated for 2 more cycles. If expected thickness was not achieved, the second round of MenSCs could be conducted. (Diagram 1)

\section{Embryo transfer}

Embryo transfer commenced when estradiol levels reached more than $200 \mathrm{pg} / \mathrm{ml}$ and progesterone less than $1 \mathrm{ng} / \mathrm{ml}$. Progesterone was used to support luteal growth. Patient returned to the hospital for pregnancy test as usual.

\section{Ultrasound measurement}

Transvaginal ultrasound examination was performed to assess the endometrial thickness. Transabdominal ultrasound was conducted to detect pregnancy outcome.

\section{Statistical analysis}

All analyses were performed using Graphpad Prism 5 software. The changes in endometrium thickness and menstrual duration were evaluated with two-tail paired Student $t$ test. $P<0.05$ was considered as statistically significant.

\section{Results}

\section{Phenotypic feature and differentiation of MenSCs}

Microscopic observations showed that MenSCs had fibroblast-like and spindle-shaped morphology (Figure 1A). Flow cytometry analysis revealed that these cells were positive for CD73, CD105, and CD90, which are typical MSCs markers, and negative for CD34, CD45, HLA-DR which are absent in MSCs ( Figure 1B).

To evaluate the differentiation capacity of MenSCs, we induced MenSCs differentiation to adipocytes and osteoblasts. The histochemical results indicated that MenSCs were able to differentiate into these two lineages (Figure 1C). The qPCR results revealed that adipogenesis-related gene LPL and osteogenesisrelated gene OC were significantly increased compared with non-induced MenSCs (Figure 1D). 


\section{Endometrium growth condition}

Enrolled patients were refractory to traditional treatment, including adhesiolysis and HRP. Their endometrium growth condition was regarded as self-control. We compared the thickness before and after MenSCs therapy; results showed that endometrium increased significantly from $3.9 \pm 0.9 \mathrm{~mm}$ to $7.5 \pm 0.6$ $\mathrm{mm}$ (Table1, Figure 2). Transvaginal ultrasound showed thicker endometrial thickness and clear morphological improvement after MenSCs treatment (Figure 3). Menstrual duration also increased from the pre-treatment $2.4 \pm 0.7$ days to the post-treatment $5.3 \pm 0.6$ days (Table 1 , Figure 2 ).

\section{Pregnancy outcomes}

The patients either tried natural conception $(\mathrm{N}=1)$ or received embryo transfers $(\mathrm{N}=11)$ when their endometrium thickness reached 7-8 mm. Five out of 12 patients conceived successfully: 1 naturally, 4 by in vitro fertilization (IVF). Up to now, four live births were achieved and 2 patients are in the second trimester.

\section{Discussion}

In the present study, a new MenSCs-based therapy was applied to treat twelve 22-40-years old women, who were diagnosed with refractory IUA and resistant to conventional treatments. Some of the patients underwent multiple IVF without success. The MenSCs were isolated and expanded in vitro successfully from all the patients. $1 \times 10^{7}$ MenSCs were transplanted into the endometrium followed by hormonal stimulation. All patients showed significant improvements in endometrial thickness and menstrual duration after the treatment. Five women achieved clinical pregnancy: 4 after embryo transfers, 1 from natural conception. These encouraging results suggest that autologous MenSCs transplantation can be a promising treatment for refractory IUA.

Various types of stem cells have been detected in the endometrium, such as epithelial progenitors, mesenchymal stem cells, side population cells and menstrual blood-derived stem cells [14-17]. MenSCs can be easily isolated and purified by adherent culture. We observed that the morphology of primary passage MenSCs is much shorter than that of the fourth passage. Once passaged, they show a typical mesenchymal characteristic with spindle fibroblast-like shape and an elongated cytoplasm, expressing mesenchymal stem cells surface markers, such as CD73, CD90, CD105 but not hematopoietic cell surface markers, such as CD34, CD45 and CD14. MenSCs exhibit the capacity to differentiate into adipocyte and osteoblasts in vitro, which was confirmed by the adipocyte-associated and osteoblast-associated genes expression. These important features are consistent with previous claims that MenSCs exhibit mesenchymal stem cells characteristic and are morphologically and functionally similar to biopsyderived endometrial cells $[18,19]$.

Currently, the predominant stem cells in the clinical research on AS is derived from bone-marrow. Nagori et al first innovatively used autologous bone marrow derived stem cells to treat a woman with severe AS, who was unsuccessfully treated with the conventional methods [20]. Her endometrium regenerated to a 
thickness of $8 \mathrm{~mm}$ and she achieved pregnancy through IVF. Subsequently, Singh et al reported 6 women with refractory AS received bone marrow mononuclear stem cells therapy. The endometrial thickness of all patients increased significantly after the treatment, and five of them regained menstruation [21]. An autologous peripheral blood CD133+ bone marrow-derived stem cells therapy in a pilot cohort study was conducted on 16 AS patients [22]. Endometrial thickness increased from an average of $4.3 \mathrm{~mm}$ to 6.7 $\mathrm{mm}$. Three patients became pregnant spontaneously and 7 pregnancies were obtained in 14 IVF cycles. These cases indicated that bone marrow derived stem cells transplantation resulted in promising effects on patients with refractory IUA. However, risk of infection, unnecessary pain and limited quantity of stem cells are the main concerns of obtaining cells from bone marrow. In the present study, we used the stem cells derived from menstrual blood due to their rapid proliferation rate and easy, noninvasive acquisition. The uses of this type of stem cells have not caused ethical controversy.

In the study by Tan et al, fresh $1 \times 10^{6}$ or $2 \times 10^{6}$ MenSCs were transplanted into the basalis two weeks after collecting menstrual blood [23]. Five out of 7 patients' endometrial thickness grew to $7 \mathrm{~mm}$. A total of 3 patients conceived. Considering that the number of the MenSCs may be a crucial factor, we increased the number of MenSCs to $1 \times 10^{7}$ in the present study. We chose the time of transplantation on the second day of the cycle in order to synchronize with menstrual cycle. We used cryopreserved cells rather than fresh cells which seemed to show the similar therapeutic efficacy. Furthermore, cryopreserved cells make clinical practice more convenient. In the present study, 10 out of 12 patients' endometrial thickness reached $7 \mathrm{~mm}$; a total of 5 patients conceived.

We speculate that there are two mechanisms for the effects of transplanted MenSCs: first, they may supplement the depleted local stem cells in AS patients; second, the paracrine effects of MenSCs could be highly beneficial. With respect to their gene expression, MenSCs are more likely to express genes in the immune and inflammatory pathway [24]. MenSCs have been used in immune and inflammation related diseases for their anti-inflammatory and immunomodulatory functions $[25,26]$. Therefore, the factors they produce could create an anti-inflammatory and immunosuppressive microenvironment that is conducive for tissue growth. However, the mechanisms need to be further explored in our future studies. What's more, large populations would be included.

\section{Conclusions}

Intrauterine transplantation of stored autologous MenSCs results in regeneration of endometrium, a prolongation of menstrual duration an increase rate of pregnancy in patients with refractory AS. Ten out of 12 patients' endometrial thickness reached $7 \mathrm{~mm}$. a total of 5 patients conceived, 4 live births achieved and 2 patients are in the second trimester.

\section{Declarations}

\section{Abbreviations}


MenSCs: Menstrual blood stem cells; IUA: intrauterine adhesion; AS: Asherman's syndrome; HRP: Hormone replacement therapy; IVF: in vitro fertilization.

\section{Acknowledgments}

Not applicable

\section{Author's contributions}

HL M came up with the ideas and conducted the transplantation of MenSCs with the help of W $W^{1}$ and LY L. KQ Y, DC W and YF L launched the collaboration between the hospital and the company. $\mathrm{HL} \mathrm{M,} \mathrm{M} \mathrm{H,}$ $M L L$ performed participant recruitment and data collection. MT $L$ wrote the first draft of the manuscript with TR D's help. MT L and W W2 isolated and cultured the MenSCs. All authors revised it critically for important intellectual content and gave final approval of the version to be published.

\section{Funding}

The study was funded by the hospital itself.

\section{Availability of data and materials}

All data generated or analyzed during this study are included in this published article.

\section{Ethics approval and consent to participate}

The study was approved by the Reproductive Medicine Ethics Committee of Guiping People's Hospital. All participants voluntarily signed a written informed consent after being fully informed of the aim, procedure and possible risks of the study.

\section{Consent for publication}

Not applicable.

\section{Competing interests}

The author(s) declared no potential conflicts of interests with respect to the research, authorship, and/or publication of this article.

\section{References}

1.

Cenksoy PO, Ficicioglu C, Yesiladali M, Kizilkale O. The diagnosis and management of Intrauterine adhesion developed after cesarean section and reproductive outcome. Case Rep Obstet Gynecol. 2013;2013:450658.

2. 
Salazar CA, Isaacson K, Morris S. A comprehensive review of Intrauterine adhesion: causes, symptoms and treatment options. Currt Opin Obstetrics Gynecol. 2017;29:249-56.

3.

Verdi J, Tan A, Shoae-Hassani A, Seifalian AM. Endometrial stem cells in regenerative medicine. J Biol Eng. 2014;8:20.

4.

Yu D, Wong YM, Cheong Y, Xia E, Li TC. Asherman syndrome-one century later. Fertil Steril. 2008;89:75979.

5.

Lee YJ, Yi KW. Bone marrow-derived stem cells contribute to regeneration of the endometrium. 2018;45:149-153.

6.

Chen L, Qu J, Xiang C. The multi-functional roles of menstrual blood-derived stem cells in regenerative medicine. Stem Cell Res Ther. 2019;10:1.

7.

Zheng Y, Lin X, Dai Y, Hu X, Zhu H, Jiang Y, et al. Endometrial stem cells repair injured endometrium and induce angiogenesis via AKT and ERK pathways. Reproduction. 2016;152:389-402.

8.

Lai D, Wang F, Yao X, Zhang Q, Wu X, Xiang C. Human endometrial mesenchymal stem cells restore ovarian function through improving the renewal of germline stem cells in a mouse model of premature ovarian failure. J Transl Med. 2015;13:155.

9.

Jiang Z, Hu X, Yu H, Xu Y, Wang L, Chen H, et al. Human endometrial stem cells confer enhanced myocardial salvage and regeneration by paracrine mechanisms. J cell Mol Med. 2013;17:1247-60. 10.

Borlongan CV, Kaneko Y, Maki M, Yu SJ, Ali M, Allickson JG, et al. Menstrual blood cells display stem celllike phenotypic markers and exert neuroprotection following transplantation in experimental stroke. Stem cells Dev. 2010;19:439-52.

11.

Lu S, Shi G, Xu X, Wang G, Lan X, Sun P, et al. Human endometrial regenerative cells alleviate carbon tetrachloride-induced acute liver injury in mice. J Transl Med. 2016;14:300.

12.

Xiang B, Chen L, Wang X, Zhao Y, Wang Y, Xiang C. Transplantation of menstrual blood-derived mesenchymal stem cells promotes the repair of LPS-induced acute lung injury. Int J Mol Sci. 2017;18:689.

13.

van der Molen RG, Schutten JH, van Cranenbroek B, ter Meer M, Donckers J, Scholten RR, et al. Menstrual blood closely resembles the uterine immune micro-enviroment and is clearly distinct from peripheral blood. Hum reprod. 2014;29:303-14.

14. 
Gargett CE, Masuda H. Adult stem cells in the endometrium. Mol Hum Reprod. 2010;16:818-34.

15.

Gargett CE, Schwab KE, Zillwood RM, Nguyen HP, Wu D. Isolation and culture of epithelial progenitors and mesenchymal stem cells from human endometrium. Bio Reprod. 2009;80:1136-45.

16.

Kato K, Yoshimoto M, Kato K, Adachi S, Yamayoshi A, Arima T, et al. Characterization of side-population cells in human normal endometrium. Hum Reprod. 2007;22:1214-23.

17.

Meng X, Ichim TE, Zhong J, Rogers A, Yin Z, Jackson J, et al. Endometrial regenerative cells: a novel stem cell population. J Transl Med. 2007;5:57.

18.

Rossignoli F, Caselli A, Grisendi G, Piccinno S, Burns JS, Murgia A, et al. Isolation, characterization, and transduction of endometrial decidual tissue multipotent mesenchymal stromal/stem cells from menstrual blood. Biomed Res Int. 2013;2013:901821.

19.

Rodrigues MC, Lippert T, Nguyen H, Kaelber S, Sanberg PR, Borlongan CV. Menstrual blood-derived stem cells: in vitro and in vivo characterization of functional effects. Adv Exp Med Biol. 2016;951:111-21. 20.

Nagori CB, Panchal SY, Patel H. Endometrial regeneration using autologous adult stem cells followed by conception by in vitro fertilization in a patient of severe Intrauterine adhesion. J Hum Reprod Sci. 2011;4:43-8.

21.

Singh N, Mohanty S, Seth T, Shankar M, Bhaskaran S, Dharmendra S. Autologous stem cell transplantation in refractory Intrauterine adhesion: A novel cell based therapy. J Hum Reprod Sci. 2014;7:93-8.

22.

Santamaria X, Cabanillas S, Cervello I, Arbona C, Raga F, Ferro J, et al. Autologous cell therapy with CD133 + bone marrow-derived stem cells for refractory Intrauterine adhesion and endometrial atrophy: a pilot cohort study. Hum Reprod. 2016;31:1087-96.

23.

Tan J, Li P, Wang Q, Li Y, Li X, Zhao D, et al. Autologous menstrual blood-derived stromal cells transplantation for severe Intrauterine adhesion. Hum Reprod. 2016;31:2723-9.

24.

Wang H, Jin P, Sabatino M, Ren J, Civini S, Bogin V, et al. Comparison of endometrial regenerative cells and bone marrow stromal cells. J Ttransl Med. 2012;10:207.

25.

Luz-Crawford P, Torres MJ, Noel D, Fernandez A, Toupet K, Alcayaga-Miranda F, et al. The immunosuppressive signature of menstrual blood mesenchymal stem cells entails opposite effects on experimental arthritis and graft versus host diseases. Stem cells. 2016;34:456-69.

26.

Page $11 / 12$ 
Leyendecker A Jr, Pinheiro CCG, Amano MT, Bueno DF. The use of human mesenchymal stem cells as therapeutic agents for the in vivo treatment of immune-related diseases: a systematic review. Front Immunol. 2018;9:2056.

\section{Tables}

Table 1.Clinical information and outcomes of patients with AS

\begin{tabular}{|c|c|c|c|c|c|c|c|c|}
\hline \multirow{2}{*}{\multicolumn{2}{|c|}{ Patient Age }} & \multirow[t]{2}{*}{$\begin{array}{l}\text { Etiology of } \\
\text { AS }\end{array}$} & \multirow[t]{2}{*}{ Prior repair attempts } & \multicolumn{2}{|c|}{$\begin{array}{l}\text { Menstrual } \\
\text { duration }\end{array}$} & \multicolumn{2}{|c|}{$\begin{array}{l}\text { Endometrial } \\
\text { thickness (mm) }\end{array}$} & \multirow[t]{2}{*}{ Pregnancy outcomes } \\
\hline & & & & Pre. & Post. & Pre. & Post. & \\
\hline 1 & 31 & Curettage & Adhesionlysis $\times 4+\mathrm{HRT}$ & 1 & 5 & 3.3 & 7.0 & No \\
\hline 2 & 34 & Curettage & Adhesionlysis $\times 3+\mathrm{HRT}$ & 2 & 5 & 3.5 & 7.5 & Yes, second trimester pregnancy \\
\hline 3 & 40 & Unexplained & Adhesionlysis $\times 3+\mathrm{HRT}$ & 3 & 6 & 3.3 & 6.8 & Yes, baby born (twins) \\
\hline 4 & 39 & Curettage & Adhesionlysis $\times 3+\mathrm{HRT}$ & 3 & 6 & 4.6 & 7.6 & Yes, baby born \\
\hline 5 & 31 & Curettage & Adhesionlysis $\times 2+\mathrm{HRT}$ & 2 & 5 & 5 & 7.2 & No \\
\hline 6 & 39 & Curettage & Adhesionlysis $\times 4+\mathrm{HRT}$ & 2 & 4 & 4.0 & 6.8 & No \\
\hline 7 & 34 & Curettage & Adhesionlysis $\times 2+\mathrm{HRT}$ & 2 & 5 & 3.5 & 7.5 & No \\
\hline 8 & 35 & Infection & Adhesionlysis $\times 2+\mathrm{HRT}$ & 3 & 6 & 4.7 & 9.0 & Yes, baby born \\
\hline 9 & 33 & Curettage & Adhesionlysis $\times 5+\mathrm{HRT}$ & 3 & 5 & 3.1 & 7.6 & $\begin{array}{l}\text { Yes, spontaneous pregnancy, } \\
\text { second trimester pregnancy }\end{array}$ \\
\hline 10 & 33 & Curettage & Adhesionlysis $\times 5+\mathrm{HRT}$ & 2 & 5 & 4.1 & 7.3 & Biochemistry pregnancy only \\
\hline 11 & 40 & Unexplained & Adhesionlysis $\times 2+\mathrm{HRT}$ & 3 & 6 & 2.2 & 8 & No \\
\hline 12 & 40 & Infection & Adhesionlysis $\times 2+\mathrm{HRT}$ & 3 & 5 & 5 & 7.5 & No \\
\hline
\end{tabular}

